\title{
Prevalencia de la depresión en España: Análisis de los últimos 15 años
}

\author{
Fernando Cardila, África Martos, Ana B. Barragán, $\mathrm{M}^{\mathrm{a}}$ del Carmen \\ Pérez-Fuentes, $\mathrm{M}^{\mathrm{a}}$ del Mar Molero y José J. Gázquez \\ Universidad de Almería (España)
}

\begin{abstract}
La depresión en España se ha convertido en los últimos años en un importante problema de salud pública que supone al Estado una alta inversión en sanidad. Los distintos trastornos depresivos han sido comúnmente asociados con otros trastornos psicopatológicos, en especial con la ansiedad, y con enfermedades físicas. Su prevalencia varía en función de las investigaciones consultadas debido a las características de las muestras, los instrumentos utilizados o el tipo de trastorno estudiado. Se realiza una revisión sistemática en distintas bases de datos nacionales e internacionales, obteniendo un total de 63 publicaciones que se ve reducido a 9 tras la aplicación de los criterios de inclusión y exclusión. Se analizan los datos concernientes a las 9 publicaciones que utilizan muestras de diversa índole: pacientes con diagnóstico de esquizofrenia, estudiantes universitarios, población clínica infantil, niños en edad preescolar, población mayor, población general, pacientes hospitalizados y fumadores. Los datos de prevalencia oscilan en los trabajos examinados desde el $1.12 \%$ en preescolares, $8.56 \%$ en población general y hasta $55.6 \%$ en estudiantes universitarios. Tras el análisis de los resultados, se observa un patrón en el género constante (con una proporción superior de mujeres que sufren depresión), aunque los datos de prevalencia varían de un trabajo a otro y su comparación resulta compleja.
\end{abstract}

Palabras clave: Revisión, prevalencia, depresión, epidemiología, trastornos mentales.

Prevalence of depression in Spain: Analysis of the last 15 years. Depression in Spain in recent years has become an important problem of public health which is the cause of heavy government healthcare spending. The various depressive disorders have commonly been associated with other psychopathological disorders, especially anxiety, and with physical diseases. Its prevalence varied depending on the study because of the characteristics of the samples, the instruments used or the type of disorder studied. A systematic review was done in several national and international databases, in which a total of 63 publications were found, with only nine remaining after applying the inclusion and exclusion criteria. The data analyzed concerning the nine publications use a diversity of samples: patients diagnosed with schizophrenia, university students, children clinical population, preschool children, elderly population, general population, hospitalized patients and smokers. The data on prevalence varied in the studies examined from $1.12 \%$ in preschool children, $8.56 \%$ in the general population and up to $55.6 \%$ in university students. After analysis of results, a constant pattern was observed in gender with a higher proportion of women who suffer from depression), although the data on prevalence varied from one study to another and their comparison was complicated.

Keywords: Review, prevalence, depression, epidemiology, mental disorders.

Correspondencia: $\mathrm{M}^{\mathrm{a}}$ del Carmen Pérez Fuentes. Departamento de Psicología. Universidad de Almería. Ctra. de Sacramento s/n. C.P.: 04120. Almería (España).E-mail: mpf421@ual.es 
En algún momento de su vida, casi un $20 \%$ de las personas residentes en España ha presentado algún trastorno mental según los criterios del DSM-IV, y un 8.4\% lo hizo en el último año. El episodio depresivo mayor es el más usual, con una prevalencia de $3.9 \%$, siendo para las mujeres más del doble que para los hombres, $5.62 \%$ frente a un 2.15\% (Haro et al., 2006). Por tanto, la probabilidad de que una persona sufra depresión a lo largo de su vida es superior a la de otros trastornos mentales como los de ansiedad o consumo de sustancias (Vindel, Salguero, West, Dongil, y Latorre, 2012). Una realidad que debe ser tenida en cuenta para evitar que las personas que padecen depresión en alguna de sus formas puedan no ser reconocidas o recibir un tratamiento inadecuado (Simon, Fleck, Lucas, y Bushnell, 2004). Además, los trastornos de tipo depresivo usualmente se dan en adición a otros tipos de desórdenes mentales (Aragonès, Piñol, y Labad, 2009) y conductas que ponen en peligro la vida del paciente, como el intento de suicidio, cuyo riesgo es tres veces superior en pacientes diagnosticados de depresión mayor en comparación con la población general (Baca y Aroca, 2014).

Estas cifras de prevalencia, sumado a las consecuencias que la depresión origina en la sociedad, ha supuesto que la depresión sea objetivo trascendental en las políticas de salud pública (Valladares, Dilla, y Sacristán, 2009), con un coste total a nivel europeo estimado en 118 billones de euros y un gasto anual de 5005 millones en el caso de España (Sobocki, Jonsson, Angst, y Rehnberg, 2006).

Existen distintos factores de riesgo que aumentan la probabilidad de una persona para presentar depresión (Frías, Carrasco, Fernández, García, y García, 2009), estos pueden ser: a) biológicos, como la predisposición genética investigada en estudios familiares (Nurnberger y Gershon, 1992), pero que cuentan con el inconveniente de no controlar los factores ambientales (Mitjans y Arias, 2012); b) cognitivo-emocionales, como el estilo atribucional depresógeno (Hankin y Abramson, 2002) o c) socioculturales, como una historia materna de depresión (Tully, Iacono, y McGue, 2008).

Por otro lado, la depresión ha sido asociada como factor de riesgo en enfermedades físicas, en especial, con enfermedades de tipo cardiovascular (Giner et al., 2014) o trastornos de tipo metabólico como la diabetes (Yu, Zhang, Lu, y Fang, 2015). La relación inversa ha sido también investigada, encontrando que pacientes hospitalizados pueden presentar sintomatología depresiva en un 21.5\% (16.3\% en hombres y $31.8 \%$ en mujeres), observándose mayores niveles de depresión y/o ansiedad en aquellos que sentían que su dolencia era de mayor gravedad (Grau, Suñer, Abulí, y Comas, 2003).

Atendiendo a distintos grupos poblacionales, se observa una prevalencia de trastornos depresivos de un $8.2 \%$ para menores entre 8 y 12 años (del Barrio, Frías, y Mestre, 1994) o de $14.6 \%$ en muestra clínica infantil menor de 19 años (Aláez, Martínez-Arias y Rodríguez, 2000). En estudiantes universitarios, los datos de prevalencia oscilan entre 37.4\% para síntomas depresivos (Arrieta, Díaz y González, 
2013), 29.9\% para depresión leve, $14.2 \%$ para depresión moderada y $3.2 \%$ para depresión severa (Gutiérrez et al, 2010). En población mayor, la prevalencia en trastornos del humor (afectivos) supone un 30.1\% del total de los trastornos mentales de los pacientes derivados a salud mental (Gázquez, Pérez, Lucas, y Yuste, 2008). En población mayor general, la prevalencia oscila en cifras entre el 16\% (García y Tobías, 2001), el 19.7\% (Urbina, Flores, García, Torres, y Torrubias, 2007) o el 34.5\% (Sarró-Maluquer, Ferrer-Feliu, Rando-Matos, Formiga, y Rojas-Farreras, 2013), entre otras.

\section{METODOLOGÍA}

\section{Unidad de análisis y búsqueda bibliográfica}

Se realizó una revisión sistemática de los estudios cuya temática se centrase en la prevalencia de la depresión en España, independientemente de cualquier otra variable. Se usaron las bases de datos ProQuest, PsycINFO, Psychology Journals, Dialnet Plus y Psicodoc. Para la búsqueda en español se usaron los descriptores "prevalencia" y "depresión" en el apartado título, y el descriptor "España" en todo el documento. Para la búsqueda de publicaciones en inglés, se usaron los descriptores "prevalence", "depression" y "Spain" siguiendo la misma fórmula. Para la búsqueda en Psicodoc se eliminó el descriptor España con el objetivo de recuperar más resultados, excluyéndose aquellas publicaciones sin participación de muestra española. El periodo se estableció desde el año 2000 hasta el año 2015. Se realizó en español y en inglés, restringiendo la búsqueda a artículos de revista. Los resultados encontrados a través de esta búsqueda quedan reflejados en la tabla 1.

Tabla 1. Bases de datos, descriptivos, fórmulas y referencias

\begin{tabular}{lccc}
\hline Base de datos & Descriptores & Fórmulas de búsqueda & $\begin{array}{c}\mathrm{N}^{\circ} \mathrm{de} \\
\text { referencias }\end{array}$ \\
\hline PsycINFO & $\begin{array}{c}\text { "Prevalence", "Depression", } \\
\text { "Spain" }\end{array}$ & $\begin{array}{c}\text { ti(prevalence) AND } \\
\text { ti(depression) AND Spain }\end{array}$ & 24 \\
\hline PsycINFO & $\begin{array}{c}\text { "Prevalencia", "Depresión", } \\
\text { "España" }\end{array}$ & $\begin{array}{c}\text { ti(prevalencia) AND } \\
\text { ti(depresión) AND España }\end{array}$ & 2 \\
\hline Dialnet Plus & $\begin{array}{c}\text { ti(prevalence) AND } \\
\text { "Spain", }\end{array}$ & 0 \\
\hline Dialnet Plus & $\begin{array}{c}\text { ti(depression) AND Spain } \\
\text { "Prevalencia", "Depresión", } \\
\text { "España" }\end{array}$ & $\begin{array}{c}\text { ti(prevalencia) AND } \\
\text { ti(depresión) AND España }\end{array}$ & 2 \\
\hline Psychology & "Prevalence", "Depression", & $\begin{array}{c}\text { ti(prevalence) AND } \\
\text { "Spain" }\end{array}$ & 13 \\
\hline $\begin{array}{l}\text { Psychology } \\
\text { Journals }\end{array}$ & "Prevalencia", "Depresión", AND Spain \\
\hline PsicoDoc & "España" & $\begin{array}{c}\text { ti(prevalencia) AND } \\
\text { ti(depresión) AND España }\end{array}$ & 0 \\
\hline PsicoDoc & "Prevalencence, "Depression", "Depresión" & $\begin{array}{c}\text { ti(prevalence) AND } \\
\text { ti(depression) }\end{array}$ & 2 \\
\hline
\end{tabular}




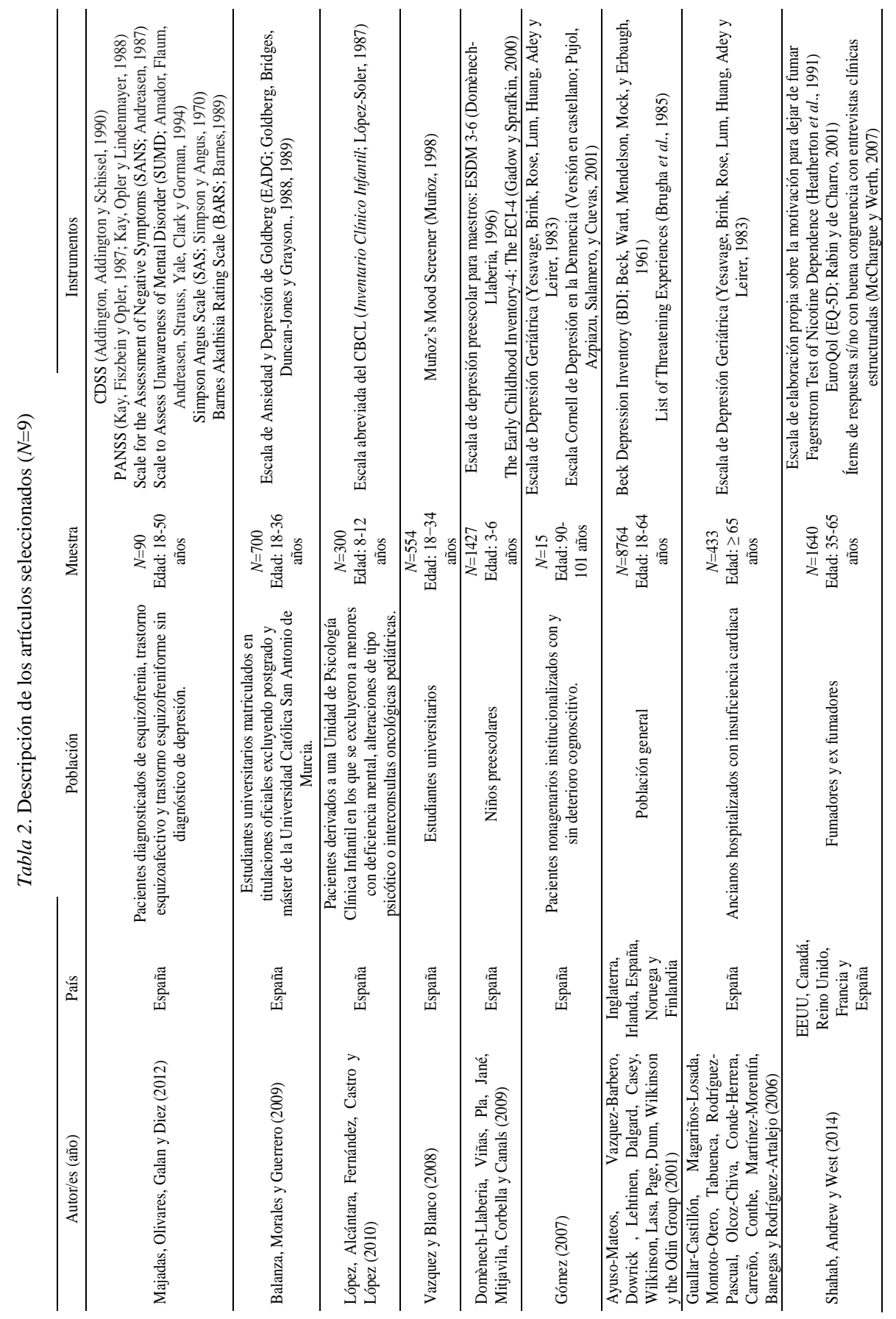


Tabla 3. Prevalencia, nosología y conclusiones

\begin{tabular}{|c|c|c|c|c|}
\hline Autor/es (año) & País & Prevalencia & Nosología & Conclusiones \\
\hline $\begin{array}{l}\text { Majadas, Olivares, } \\
\text { Galan, y Diez (2012) }\end{array}$ & España & $31 \%$ & DSM-IV-TR & $\begin{array}{c}\text { Asociada a mayor grado de severidad de la } \\
\text { enfermedad. }\end{array}$ \\
\hline $\begin{array}{l}\text { Balanza, Morales, y } \\
\text { Guerrero (2009) }\end{array}$ & España & $55.6 \%$ & DSM-IV-TR & $\begin{array}{l}\text { Algunos de los factores de riesgo para padecer } \\
\text { depresión son los largos desplazamientos diarios a la } \\
\text { universidad y las obligaciones extra-académicas de } \\
\text { tipo laboral o familiar. La aparición de los trastornos } \\
\text { suele además, ir acompañada de procesos de } \\
\text { desadaptación al entorno cercano. }\end{array}$ \\
\hline $\begin{array}{l}\text { López, Alcántara, } \\
\text { Fernández, Castro, y } \\
\text { López (2010) }\end{array}$ & España & $32 \%$ & $\begin{array}{l}\text { Factores } \\
\text { CBCL-DSM }\end{array}$ & $\begin{array}{l}\text { Más de la mitad de los menores derivados a consulta } \\
\text { presentan problemas de ansiedad, y un tercio, } \\
\text { problemas afectivos y quejas somáticas. El número } \\
\text { de niños con problemas clínicos en edad escolar es } \\
\text { mayor que de niñas. }\end{array}$ \\
\hline $\begin{array}{l}\text { Vázquez y Blanco } \\
\text { (2008) }\end{array}$ & España & $8.7 \%$ & $\begin{array}{l}\text { DSM-IV } \\
\text { (Episodio } \\
\text { Depresivo } \\
\text { Mayor) }\end{array}$ & $\begin{array}{c}\text { Necesidad de desarrollo de programas específicos } \\
\text { para estudiantes universitarios con el propósito de } \\
\text { prevenir la depresión y mejorar la adaptación a la } \\
\text { vida universitaria. }\end{array}$ \\
\hline $\begin{array}{l}\text { Domènech-Llaberia et } \\
\text { al. (2009) }\end{array}$ & España & $1.12 \%$ & $\begin{array}{l}\text { DSM-IV } \\
\text { (Trastorno } \\
\text { Depresivo } \\
\text { Mayor) }\end{array}$ & $\begin{array}{l}\text { La mayoría de los niños en edad preescolar no son } \\
\text { derivados para su diagnóstico y tratamiento. La } \\
\text { detección y el tratamiento de síntomas depresivos } \\
\text { pueden evitar la continuidad y recaídas comunes en } \\
\text { los desórdenes emocionales. }\end{array}$ \\
\hline Gómez (2007) & España & $60 \%$ & DSM-IV-TR & $\begin{array}{l}\text { Se observa comorbilidad entre depresión y deterioro } \\
\text { funcional, lo que hace necesario un plan integral de } \\
\text { cuidados que atienda a todos los déficits del anciano. }\end{array}$ \\
\hline $\begin{array}{l}\text { Ayuso-Mateos et al. } \\
(2001)\end{array}$ & $\begin{array}{l}\text { Inglaterra, } \\
\text { Irlanda, España, } \\
\text { Noruega y } \\
\text { Finlandia }\end{array}$ & $8.56 \%$ & CIE-10 & $\begin{array}{c}\text { Una substancial proporción de población en edad } \\
\text { laboral tiene un trastorno depresivo que podría verse } \\
\text { mejorado si se realizase una intervención en el corto } \\
\text { plazo. } \\
\text { Las zonas rurales muestran menos prevalencia de } \\
\text { depresión que las zonas urbanas. }\end{array}$ \\
\hline $\begin{array}{l}\text { Guallar-Castillón et al. } \\
\text { (2006) }\end{array}$ & España & $48.5 \%$ & CIE-10/DSM & $\begin{array}{c}\text { La depresión y la insuficiencia cardíaca tienen dos } \\
\text { mecanismos de asociación:biológicos (activación } \\
\text { neurohormonal entre otros) y psicosociales (la } \\
\text { depresión aumenta el incumplimiento en el } \\
\text { tratamiento y el apoyo social). } \\
\text { La depresión es más frecuente en pacientes con } \\
\text { grado funcional bajo. }\end{array}$ \\
\hline $\begin{array}{l}\text { Shahab, Andrew, y } \\
\text { West (2014) }\end{array}$ & $\begin{array}{l}\text { EEUU, Canadá, } \\
\text { Reino Unido, } \\
\text { Francia y } \\
\text { España }\end{array}$ & $22.3 \%$ & - & $\begin{array}{l}\text { Dejar de fumar mejora la salud mental en población } \\
\text { general y no exacerba síntomas depresivos en } \\
\text { personas con historia de depresión. }\end{array}$ \\
\hline
\end{tabular}

\section{Proceso de selección de las publicaciones}

Se encontraron un total de 63 publicaciones. En primer lugar, se procedió a la eliminación de los trabajos duplicados, descartando 10 de ellos. Se establecieron unos criterios de inclusión y exclusión de aplicación en los abstracts y en el texto completo. Los criterios de inclusión fueron: a) Estudios que establezcan la prevalencia de la depresión en distintas poblaciones; b) Con cualquier metodología; c) Sin limitaciones de edad o sexo; d) Con acceso al texto completo; e) Escritos en español e inglés. Los criterios de exclusión fueron los siguientes: a) Trabajos teóricos, notas editoriales o 
estudios de caso único; b) Trabajos que no aporten datos de prevalencia de la depresión; c) Trabajos cuya muestra o parte de ella no se tome en territorio español.

Tras la aplicación de los criterios de inclusión/exclusión, se eliminaron 22 referencias por no tener acceso al texto completo, 5 por tratarse de trabajos de tipo teórico, 1 por no ofrecer datos de prevalencia de depresión, 16 por no incluir una muestra de residentes en España. Se eliminó además, el estudio de Casey et al. (2008), ya que los datos presentados se referían a la prevalencia de ideación suicida en lugar de a la prevalencia de la depresión, que sí se recogían en un artículo relacionado que utiliza los mismos datos referentes a la muestra y que fue incluido en su lugar. Finalmente, los trabajos incluidos en la revisión son 9. Estos se muestran en la tabla 2, completando la información relativa a la nosología, prevalencia y conclusiones aportada por los mismos en la tabla 3.

\section{RESULTADOS}

Tras el análisis de las publicaciones seleccionadas, se observa una gran diversidad en la población objetivo sobre la que se pretende estudiar la prevalencia de la depresión. Los trabajos contemplan edades que van desde la edad preescolar (de tres a seis años) hasta la vejez. Y se incluyen muestras con características muy específicas, como estudiantes universitarios o pacientes esquizofrénicos.

Esto dificulta la comparación de los datos que arrojan los estudios acerca de la prevalencia de la depresión, pues las distintas muestras son muy heterogéneas y la variabilidad en los resultados es esperable. No obstante, esta circunstancia cuenta con el beneficio de conseguir una imagen global, variada y compleja de la depresión y su prevalencia en distintos segmentos de la sociedad.

\section{Prevalencia de depresión en pacientes con problemas psicopatológicos}

previos

La depresión a menudo se da junto con otros tipos de trastornos mentales, Majadas, Olivares, Galán, y Diez (2012) encontraron una prevalencia de un $31 \%$ en una muestra de pacientes con diagnóstico de esquizofrenia, trastorno esquizoafectivo o trastorno esquizofreniforme de acuerdo al Manual Diagnóstico y Estadístico de los Trastornos Mentales (DSM IV-TR).

En pacientes con dependencia tabáquica, Shahab, Andrew, y West (2014) hallaron una prevalencia del $22.3 \%$. Cifra por debajo de otros trastornos como la ansiedad, en la que se obtuvo una prevalencia de $27.4 \%$, pero que es consistente con los datos que se han obtenido en estudios similares (McClave et al., 2009).

La prevalencia de problemas afectivos en una muestra clínica de 300 menores resultó del 32\% (López, Alcántara, Fernández, Castro, y López, 2010). Dicha muestra se 
conformó de pacientes derivados de distintas unidades y de interconsultas de atención primaria. Es importante detenerse en esta cuestión, pues las características de esta muestra difieren de las de la población general, razón por la que la prevalencia de trastornos afectivos puede ser tan alta.

\section{Prevalencia de depresión en función de la edad}

Todos los trabajos consultados hacen, en mayor o menor medida, referencia a la edad, siendo algunos de ellos específicos de un segmento en concreto. En edad preescolar (entre 3 y 6 años), la prevalencia de depresión mayor es de $1.12 \%$, con un promedio entre niños y niñas similar y que aumenta según avanza la edad (Domènech-Llaberia et al., 2009), incrementándose la cifra hasta el $4.2 \%$ en adolescentes de 13 a 15 años (Domènech-Llaberia, Subirà, y Cuxart, 1996).

Anteriormente se ha comentado la prevalencia del $32 \%$ en menores de entre 8 y 12 años que conformaron una muestra clínica (López et al., 2010) y que, por tanto, no se puede comparar con la población general. Los autores examinaron esta cuestión y calcularon cual sería la prevalencia teniendo en consideración las diferencias entre muestra y población general, obteniendo unas cifras entre el $13.5 \%$ y el $17.9 \%$. Resultados similares a los obtenidos en otros estudios en los que se encontró que niños y niñas que sufrían depresión eran de $17.21 \%$ y $15 \%$ respectivamente (López-Soler y López-Pina, 1998) pero que distan del 3.8\% encontrado por Jaureguizar, Bernaras, Soroa, Sarasa, y Garaigordobil (2015) y del 4\% que la OMS (2005) informó en menores de entre 12 y 17 años.

En estudiantes universitarios, se ha establecido una prevalencia del $55.6 \%$ para trastornos depresivos (Balanza, Morales, y Guerrero, 2009), y del 8.7\% para el episodio depresivo mayor, siendo en el $43.8 \%$ de los afectados su primer episodio depresivo a los 18 años o más (Vázquez y Blanco, 2008).

Ayuso et al. (2001) hallaron una prevalencia de un $8.56 \%$ en una muestra $(N=8764)$ internacional, con una prevalencia específica para la muestra española $(N=1250)$ de un $2.6 \%$ (la más baja de todas las zonas estudiadas). Aunque los autores no incluyeron los resultados por grupos de edad, la distribución de la muestra española fue la siguiente: grupo de 18-25 años (20.4\%), grupo de 26-45 años (42.9\%) y grupo de 46-65 años (36.6\%).

A medida que aumenta la edad, se produce un incremento de las enfermedades ligadas a ésta (Rodríguez y Sánchez, 2004), entre las que se encuentran las insuficiencias cardiacas. Guallar-Castillón et al. (2006) incluyeron esta característica para estudiar la prevalencia de la depresión en pacientes hospitalizados de más de 65 años, obteniendo que el $48.5 \%$ de ellos manifestaba depresión.

En pacientes nonagenarios institucionalizados, se ha encontrado una prevalencia de un $60 \%$, una cifra muy alta pero que debe ser tomada con prudencia, ya 
que la muestra del estudio es pequeña y la mayoría de los pacientes padecían deterioro cognitivo con diagnósticos de demencias, enfermedad de Alzheimer, demencia mixta, demencia vascular y demencia no clasificable (Gómez, 2007).

\section{Prevalencia de depresión en función del sexo}

En edad preescolar, la prevalencia de depresión es la misma en el género masculino y femenino (Domènech-Llaberia et al., 2009). Para menores de entre 8 y 12 años en atención psicológica, la distribución por sexo resultó en un mayor número de niños con problemas afectivos, pero esto pudo ser debido a que la muestra estuvo compuesta por más del doble de niños que de niñas (López et al., 2010). Para estudiantes universitarios, se encontró una prevalencia en mujeres del $10.4 \%$ para el Episodio Depresivo Mayor, mientras que en hombres descendía al 5.3\% (Vázquez y Blanco, 2008). En otro estudio también con estudiantes universitarios, la prevalencia de trastornos depresivos difiere entre hombres y mujeres, obteniendo un $51.4 \%$ y un $60.5 \%$ respectivamente (Balanza, Morales, y Guerrero, 2009).

Ayuso-Mateos et al. (2001) obtuvieron una prevalencia del $8.56 \%$ con una distribución entre sexos de $10.05 \%$ para las mujeres y $6.61 \%$ para los hombres en la muestra total, y de $2.6 \%$ para las mujeres y $2.5 \%$ para los hombres en la muestra española.

En pacientes hospitalizados con insuficiencia cardiaca de más de 65 años, se encontró que un $57 \%$ de las mujeres y un $37.6 \%$ de los hombres presentaba depresión (Guallar-Castillón et al., 2006).

\section{CONCLUSIÓN}

La comparación a lo largo del presente trabajo de las cifras obtenidas en los distintos estudios resulta complicada por distintos motivos. Por lo general, no se encuentran dos estudios en los que se mida la depresión con el mismo instrumento, de hecho, se han encontrado distintas formas de definir y tratar el constructo, ya sea como problema, trastorno, desorden o distintas entidades nosológicas del Manual Diagnóstico y Estadístico de los Trastornos Mentales (DSM) o de la Clasificación Internacional de Enfermedades (CIE).

En la Unión Europea, el diagnóstico de depresión mayor se ha visto mantenido en el tiempo en los últimos años, la estimación de prevalencia de dicho trastorno en 2005 fue idéntica en 2011, del 6.9\% (Wittchen, 2011). Una cifra inferior a todos los resultados encontrados en las publicaciones analizadas, incluyendo aquellas que tienen muestra de diversos países. En ocasiones, las personas cercanas creen que una persona puede estar deprimida sin estarlo realmente, en el estudio de Domènech-Llaberia et al. (2009) se encontró que la prevalencia de depresión en niños preescolares era de un 
$15 \%$ con las respuestas que daban sus docentes, mientras que los diagnosticados suponían el $1.12 \%$ del total. Cabe preguntarse si existía un infradiagnóstico en la muestra debido a que, tal y como indican los autores del estudio, la mayoría de niños y niñas preescolares con depresión no son remitidos a los servicios de salud mental para su evaluación y tratamiento. Se debe destacar también, la posibilidad de que existiese una tendencia en los maestros de ver sintomatología fantasma, algo que no contemplan los autores en su estudio. En su lugar, se centran en resaltar la utilidad de un buen instrumento de screening para menores de 6 años que detecte a aquellos en riesgo de sufrir depresión.

La prevalencia en población clínica infantil encontrada por Domènech-Llaberia et al. (2009) coincide con la de otros estudios similares (Aláez, Martínez-Arias, y Rodríguez, 2000). En estudiantes universitarios, los resultados de las publicaciones analizadas muestran cifras de prevalencia muy altas en comparación con otros estudios (Arrieta, Díaz, y González, 2013; Gutiérrez et al, 2010) que, aun mostrando cifras que llegan hasta el $37.4 \%$, difiere del $55.6 \%$ aportada por Balanza, Morales, y Guerrero (2009). Para población general, Ayuso-Mateos et al. (2001) hallaron una prevalencia del 8.56\%, coherente con el 8.4\% de Haro et al. (2006).

En población anciana, la prevalencia de la depresión y trastornos relacionados (como la distimia) supone un porcentaje elevado de los trastornos mentales que, además, es mayor para el género femenino en comparación con los hombres (Gázquez et al., 2008). Tendencia que se repite en todos los estudios analizados para todos los grupos de edad a excepción del estudio de Domènech-Llaberia et al. (2009), en los que la prevalencia para niños fue mayor que para las niñas. No obstante, se debe destacar que la muestra estaba formada por 207 niños y 93 niñas que eran derivados a la Unidad de Psicología Clínica del Hospital Universitario Virgen de la Arrixaca.

La prevalencia que se desprende de los resultados del estudio seleccionado de Guallar-Castillón et al. (2006) en población de más de 65 años es del 48.5\%, unos números bastante altos si se comparan con otros estudios al respecto: 16\% (García, Tobías, 2001), 19.7\% (Urbina et al., 2007) o 34.5\% (Sarró-Maluquer et al., 2013). Se debe añadir no obstante, que la muestra del estudio de Guallar-Castillón poseía la particularidad de estar hospitalizada, lo que puede afectar al incremento de sintomatología depresiva (Grau et al., 2003).

Los datos revisados llevan a destacar la dispersión en las cifras de prevalencia. Como se ha expuesto anteriormente, las diferentes tipologías de muestras, los instrumentos que varían según el estudio, el tratamiento que se hace del término depresión o el tipo de diseño del estudio pueden ser causas de la alta variabilidad de los resultados. Cabría preguntarse, como propósito para futuras investigaciones, si existen causas distintas a las ya propuestas por las que la prevalencia de la depresión fluctúa de forma tan acentuada, como el momento temporal de realización del estudio y si coincidía 
o no con algún evento destacable (desastres naturales) o estación del año. Las diferencias socio-culturales entre distintas regiones geográficas, incluso del mismo país, podrían provocar también variabilidad en los resultados que, de obviarlas, podrían llevar a conclusiones erróneas.

\section{REFERENCIAS}

Addington, D., Addington, J., y Schissel B. (1990). A depression rating scale for schizophrenics. Schizophrenia Research, 3(4), 247-251.

Aláez, M., Martínez-Arias, R., y Rodríguez, C. (2000). La prevalencia de trastornos psicológicos en niños y adolescentes, su relación con la edad y el género. Psicothema, 12(4), 525-532.

Amador, X.F., Flaum, M., Andreasen, N.C., Strauss, D.H., Yale, S.A., Clark, S.C., y Gorman, J.M. (1994). Awareness of illness in schizophrenia and schizoaffective and mood disorders. Archives of General Psychiatry, 51(10), 826-836.

Andreasen, N.C. (1989). The Scale for the Assessment of Negative Symptoms (SANS): conceptual and theoretical foundations. The British Journal of Psychiatry, 1(7), 49-58.

Aragonès, E., Piñol, J.L., y Labad, A. (2009). Comorbilidad de la depresión mayor con otros trastornos mentales comunes en pacientes de atención primaria. Atención primaria, 41(10), 545-551.

Arrieta, K., Díaz, S., y González, F. (2013). Síntomas de depresión, ansiedad y estrés en estudiantes de odontología: prevalencia y factores relacionados. Revista Colombiana de Psiquiatría, 42(2), 173-181.

Ayuso-Mateos, J.L., Vazquez-Barbero, J.L., Dowrick, C., Lehtinen, V., Dalgard, O.S., Casey, C., Wilkinson, C., Lasa, L., Page, H., Dunn, G., Wilkinson, G., y the Odin Group (2001). Depressive disorders in Europe: prevalence figures from the ODIN study. British Journal of Psychiatry, 179(4), 308-316.

Baca, E., y Aroca, F. (2014). Factores de riesgo de la conducta suicida asociados a trastornos depresivos y ansiedad. Salud Mental, 37(5), 373-380.

Balanza, S., Morales, I., y Guerrero, J. (2009). Prevalencia de ansiedad y depresión en una población de estudiantes universitarios: factores académicos y sociofamiliares asociados. Clínica y Salud, 20(2), 177-187.

Barnes, T.R. (1989). A rating scale for drug-induced akathisia. The British Journal of Psychiatry, 154(5), 672-676.

Beck, A.T., Ward, C. H., Mendelson, M., Mock, J., y Erbaugh, J. (1961). An inventory for measuring depression. Archives of General Psychiatry, 4(6), 561-571.

Brugha, T., Bebbington, P., Tennant, C., y Hurry, J. (1985). The List of Threatening Experiences: a subset of 12 life event categories with considerable long-term contextual threat. Psychological Medicine, 15(1), 189-194.

Casey, P., Dunn, G., Kelly, B.D, Lehtinen, V., Dalgard, O.S., Dowrick, C., y Ayuso-Mateos, J.L. (2008). The prevalence of suicidal ideation in the general population: results from the Outcome of Depression International Network (ODIN) study. Social Psychiatry and Psychiatric Epidemiology, 43(4), 299-304.

Del Barrio, V., Frías, D., y Mestre, V. (1994). Autoestima y depresión en niños. Revista de Psicología General y Aplicada, 47(4), 471-476.

Domènech-Llaberia, E., Subirà, S., y Cuxart, F. (1996). Trastornos del estado de ánimo en la adolescencia temprana. En: J. Buendía (Ed.) Psicopatología en niños y adolescentes, desarrollos actuales (pp. 265-277). Madrid: Pirámide. 
Domènech-Llaberia, E. (1996). Escala de depresión infantil para maestros de preescolar: ESDM3-6. Manuscrito no publicado. Universidad Autónoma de Barcelona. Unidad de Psicopatología Infantil.

Domènech-Llaberia, E., Viñas, F., Pla, E., Jané, M.C., Mitjavila, M., Corbella, T., y Canals, J. (2009). Prevalence of major depression in preschool children. European Child \& Adolescent Psychiatry, 18, 597-604.

Frías, A., Carrasco, A., Fernández, M.J., García, A., y García, B. (2009). Comorbilidad entre trastornos de conducta y depresión mayor en la adolescencia: prevalencia, teorías explicativas y estatus nosológico. Revista de Psicopatología y Psicología Clínica, 14(1), $1-16$.

Gadow, K.D., y Sprafkin, J. (2000). Early Childhood Inventory-4 screening manual. New York: Checkmate Plus, Stony Brook.

García, M.J., y Tobías, J. (2001). Prevalencia de depresión en mayores de 65 años. Perfil del anciano de riesgo. Atención Primaria, 27(7), 484-488.

Gázquez, J.J., Pérez-Fuentes, M.C., Lucas, F., y Yuste, N. (2008). Prevalencia de los trastornos mentales en la población mayor. Anales de Psicología, 24(2), 327-333.

Giner, J., Saiz, J., Bobes, J., Zamorano, E., López, F., Hernando, T., Rico-Villademoros, F., Álamo, C., Cervilla, J.A., Ibáñez, A., Ibáñez, E., López, S., Morán, P., Palao, D.J., y Romacho, M. (2014). Consenso español de salud física del paciente con depresión. Revista de Psiquiatría y Salud Mental, 7(4), 195-207.

Goldberg, D., Bridges, K., Duncan-Jones, P., y Grayson, D. (1988). Detecting anxiety and depression in general medical settings. British Medical Journal, 97, 897-899.

Goldberg, D., Bridges, K., Duncan-Jones, P., y Grayson, D. (1989). Detección de la ansiedad y la depresión en el marco de la medicina general. British Medical Journal, 4(2), 49-53.

Gómez, P.S. (2007). Prevalencia de depresión en pacientes nonagenarios institucionalizados. Informaciones psiquiátricas: publicación científica de los Centros de la Congregación de Hermanas Hospitalarias del Sagrado Corazón de Jesús, 188, 189-196.

Grau, A., Suñer, R., Abulí, P., y Comas, P. (2003). Niveles de ansiedad y depresión en enfermos hospitalizados y su relación con la gravedad de la enfermedad. Medicina Clínica, 120(10), 370-375.

Guallar-Castillón, P., Magariños-Losada, M.M., Montoto-Otero, C., Tabuenca, A.I., RodríguezPascual, C., Olcoz-Chiva, M., Conde-Herrera, M., Carreño, C., Conthe, P., MartínezMorentín, E., Banegas, J.R., y Rodríguez-Artalejo, F. (2006). Prevalencia de depresión, y factores biomédicos y psicosociales asociados, en ancianos hospitalizados con insuficiencia cardiaca en España. Revista Española de Cardiología, 59(8), 770-778.

Gutiérrez, J.A., Montoya, L.P., Toro, B.E., Briñón, M.A., Rosas, E., y Salazar, L. (2010). Depresión en estudiantes universitarios y su asociación con el estrés académico. Revista CES Medicina, 24(1), 7-17.

Hankin, B.L., y Abramson, L.Y. (2002). Measuring cognitive vulnerability to depression in adolescents: Reliability, validity, and gender differences. Journal of Clinical Child and Adolescent Psychology, 31(4), 491-504.

Haro, J.M., Palacín, C., Vilagut, G., Martínez, M., Bernal, M., Luque, I., Codony, M., Dolz, M., Alonso, J., y el Grupo ESEMeD-España (2006). Prevalencia de los trastornos mentales y factores asociados: resultados del estudio ESEMeD-España. Medicina Clínica, 126(12), 445-451.

Heatherton, T.F., Kozlowski, L.T., Frecker, R.C., y Fagerström, K.O. (1991). The Fagerström Test for Nicotine Dependence: a revision of the Fagerström Tolerance Questionnaire. British Journal of Addiction, 86(9), 1119-1127. 
Jaureguizar, J., Bernaras, E., Soroa, M., Sarasa, M., y Garaigordobil, M. (2015). Sintomatología depresiva en adolescentes y variables asociadas al contexto escolar y clínico. Psicología Conductual, 23(2), 245-264.

Kay, S.R., Fiszbein, A., y Opler, L.A. (1987). The positive and negative syndrome scale (PANSS) for schizophrenia. Schizophrenia Bulletin, 13(2), 261-276.

Kay, S.R., Opler, L.A., y Lindenmayer, J.P. (1988). Reliability and validity of the positive and negative syndrome scale for schizophrenics. Psychiatry Research, 23(1), 99-110.

López, C., Alcántara, M.V., Fernández, V., Castro, M., y López, J.A. (2010). Características y prevalencia de los problemas de ansiedad, depresión y quejas somáticas en una muestra clínica infantil de 8 a 12 años, mediante el CBCL (Child Behavior Checklist). Anales de Psicología, 26(2), 325-334.

López-Soler, C. (1987). Inventario de Conductas Infantiles. Manuscrito no publicado.

Majadas, S., Olivares, J., Galán, J., y Díez, T. (2012). Prevalence of depression and its relationship with other clinical characteristics in a sample of patients with stable schizophrenia. Comprehensive Psychiatry, 53(2), 145-151.

McChargue, D.E., y Werth, C.J. (2007). Depression vulnerability within smoking research: how accurate are one-item screening items? Addictive Behaviors, 32(2), 404-409.

McClave, A.K., Dube, S.R., Strine, T.W., Kroenke, K., Caraballo, R.S., y Mokdad, A.H. (2009). Associations between smoking cessation and anxiety and depression among U.S. adults. Addictive Behaviors, 34, 491-497.

Mitjans, M., y Arias, B. (2012). La genética de la depresión: ¿qué información aportan las nuevas aproximaciones metodológicas? Actas Españolas de Psiquiatría, 40(2), 70-83.

Muñoz, RF. (1998). Preventing major depression by promoting emotion regulation: a conceptual framework and some practical tools. International Journal of Mental Health Promotion, l(1), 23-40.

Nurnberger, J.I., y Gershon, E.S. (1992). Genetics. En: E.S. Paykel (Ed.), Handbook of Affective Disorders, $2^{a}$ Edición (pp. 219-253). London: Guilford Press.

Pujol, J., Azpiazu, P., Salamero, M., y Cuevas, R. (2001). Sintomatología depresiva en la demencia. Escala de Cornell: validación de la versión en castellano. Revista de Neurología, 33(4), 397-398.

Rabin, R., y de Charro, F. (2001). EQ-5D: a measure of health status from the EuroQol Group. Annals of Medicine, 33(5), 337-343.

Rodríguez, M., y Sánchez, J.L. (2004). Reserva cognitiva y demencia. Anales de Psicología, 20(2), 175-186.

Sarró-Maluquer, M., Ferrer-Feliu, A., Rando-Matos, Y., Formiga, F., y Rojas-Farreras, S. (2013). Depresión en ancianos: prevalencia y factores asociados. Semergen, 39(7), 354-360.

Shahab, L., Andrew, S., y West, R. (2014). Changes in prevalence of depression and anxiety following smoking cessation: results from an international cohort study (ATTEMPT). Psychological Medicine, 44(1), 127-141.

Simon, G.E., Fleck, M., Lucas, R., y Bushnell, D.M. (2004). Prevalence and Predictors of Depression Treatment in an International Primary Care Study. The American Journal of Psichiatry, 161(9), 1626-1634.

Simpson, G.M., y Angus, J.W. (1970). A rating scale for extrapyramidal side effects. Acta Psychiatrica Scandinavica, 45(212), 11-19.

Sobocki, P., Jonsson, B., Angst, J., y Rehnberg, C. (2006). Cost of depression in Europe. The Journal of Mental Health Policy and Economics, 9(2), 87-98.

Tully, E.C., Iacono, W.G., y McGue, M. (2008). An adoption study of parental depression as an environmental liability for adolescent depression and childhood disruptive disorders. American Journal of Psychiatry, 165(9), 1148-1154. 
Urbina, J.R., Flores, J.M., García, M.P., Torres, L., y Torrubias, R.M. (2007). Síntomas depresivos en personas mayores. Prevalencia y factores asociados. Gaceta Sanitaria, 21(1), 37-42.

Valladares, A., Dilla, T., y Sacristán, J.A. (2009). La depresión: una hipoteca social. Últimos avances en el conocimiento del coste de la enfermedad. Actas Españolas de Psiquiatría, $37(1), 49-53$.

Vázquez, F.L., y Blanco, V. (2008). Prevalence of DSM-IV Major Depression Among Spanish University Students. Journal of American College Health, 57(2), 165-171.

Vindel, A.C., Salguero, J.M., West, C.M., Dongil, E., y Latorre, J.M. (2012). La depresión en atención primaria: prevalencia, diagnóstico y tratamiento. Papeles del Psicólogo, 33(1), $2-11$.

Wittchen, H.U., Jacobi, F., Rehm, J., Gustavsson, A., Svensson, M., Jönsson, B., Olesen, J., Allgulander, C., Alonso, J., Faravelli, C., Fratiglioni, L., Jennum, P., Lieb, R., Maercker, A., van Os, J., Preisig, M., Salvador-Carulla, L., Simon, R., y Steinhausen, H.-C. (2011). The size and burden of mental disorders and other disorders of the brain in Europe 2010. European Neuropsychopharmacology, 21(9), 655-679.

Yesavage, J.A., Brink, T.L., Rose, T.L., Lum, O., Huang, V., Adey, M., y Leirer, V.O. (1983). Development and validation of a geriatric depression rating scale: a preliminary report. Journal of Psychiatry Research, 17(1), 37-49.

Yu, M., Zhang, X., Lu, F., y Fang, L. (2015). Depression and risk for diabetes: a meta-analysis. Canadian Journal of Diabetes, 39(4), 266-272.

Recibido: 10 de mayo de 2015

Recepción Modificaciones: 1 de junio de 2015

Aceptado: 15 de junio de 2015 